\title{
1 Molecular Squares, Boxes, and Cubes
}

\author{
Peter H. Dinolfo \\ Shih-Sheng Sun \\ Joseph T. Hupp \\ Northwestern University, Evanston, Ilinois, U.S.A.
}

\section{INTRODUCTION}

Molecular squares, boxes, and cubes featuring transition- 53 metal corners are the focus of tremendous activity in 54 contemporary synthetic coordination chemistry. Their 55 design and study comprise a promising subfield of 56 inorganic and organometallic supramolecular chemistry. 57 The interest in these objects is partly aesthetic: they are 58 beautiful high-symmetry assemblies. The interest is also 59 functional: the assemblies contain cavities that are 60 capable, in principle, of encapsulating, and then sensing, 61 processing, or transporting useful molecules or atomic 62 ions - in other words, all the functions associated with 63 organic host-guest chemistry.

Why coordination chemistry? And why discrete mole- 65 cules? Actually, metal-free squares and boxes exist, albeit, 66 without the ubiquity of the metal-containing systems. 67 Notable examples include the various viologen-derived 68 cyclophanes or boxes of Stoddart and coworkers, ${ }^{[1]}$ — clear 69 antecedents of many molecular squares. What metal ions offer are a readily accessible range of angles for ligandmetal-ligand subunits (including right angles, which is 70 difficult to achieve with carbon chemistry), structural predictability based on well-known metal-ion coordination 71 motifs (linear, octahedral, square planar, etc.), a range of charges, a range of ligand-binding capacities, and, perhaps 72 most importantly, a propensity to engage in highly 73 efficient directed assembly. Metals, of course, can also 74 usefully expand the range of redox, photophysical, and 75 catalytic properties displayed by supramolecular assem- 76 blies. In principle, squares and other structures can be 77 assembled not only as discrete molecules, but also as 78 porous two- and three-dimensional arrays or coordination 79 polymers. While the array strategy clearly works ${ }^{[2]}$ a 80 common complication is the formation of interpenetrating 81 grids that eliminate most of the void volume associated 82 with isolated cavities. Preassembly of discrete squares 83 and related structures avoids the problem; indeed, to date 84 there are no examples of extended catenation, the 85 molecular equivalent of grid interpenetration.

An important idea suggested by the chemistry of 87 coordination polymers is higher-order assembly of squares 88 and other structures into large void-volume, high-porosity 89 molecular materials. This can be done surprisingly easily using van der Waals interactions (which, of course, are large for large molecules), although more elaborate and controllable strategies are emerging. In any case, the notion of porous molecular materials and the stacking of cavities to make uniformly sized channels is one that is receiving increasing attention as supramolecular coordination chemistry evolves from a mainly synthetic effort toward one that also strongly focuses on function.

Finally, the connection to nanotechnology cannot be overlooked. The cavity sizes of existing molecular squares, boxes, and cubes extend from about 0.4-5 nm, as measured along the cavity edges. These, of course, are the right dimensions to couple to other nanoscale objects or simply to function as building blocks for periodically nanostructured thin films or other materials. In these contexts, descriptions of functional suparamolecular assemblies as nanoreactors, nanofactories, nanogates, nanotemplates, and so on, are appropriate.

\section{MOLECULAR SQUARES}

\section{Some Early Examples}

The first metal-containing molecular squares appeared in 1983. ${ }^{[3]}$ They featured $\mathrm{M}(\mathrm{CO})_{4}(M=\mathrm{Cr}$, W, or $\mathrm{Mo})$ corners and $\mathrm{P}\left(\mathrm{OCH}_{2}\right)_{3} \mathrm{P}$ ligand edges, 1 (Fig. 1). While these compounds are potentially capable of behaving as hosts for small molecular guests, interest in squares as receptors or hosts really did not take hold until 1990 when an ethylenediamine $\mathrm{Pd}(\mathrm{II})$ square featuring 4,4'-bipyridyl edges, 2, was shown to function as a hydrophobic host for organic guests in water as solvent. ${ }^{[4,5]}$ Aqueous solubility was engendered by the square's $8+$ charge and by the use of nitrate as a counterion. Nuclear magnetic resonance (NMR) titrations revealed association constants on the order of $10^{2}$ for planar, electron-rich aromatic species, while aliphatic compounds showed little propensity to associate. The difference was attributed to the ability of the electron-rich, aromatic guests to form charge-transfer complexes with the bridging bipyridine ligands of the molecular square. Extension of the chemistry to $\mathrm{Pt}$ (II) was 

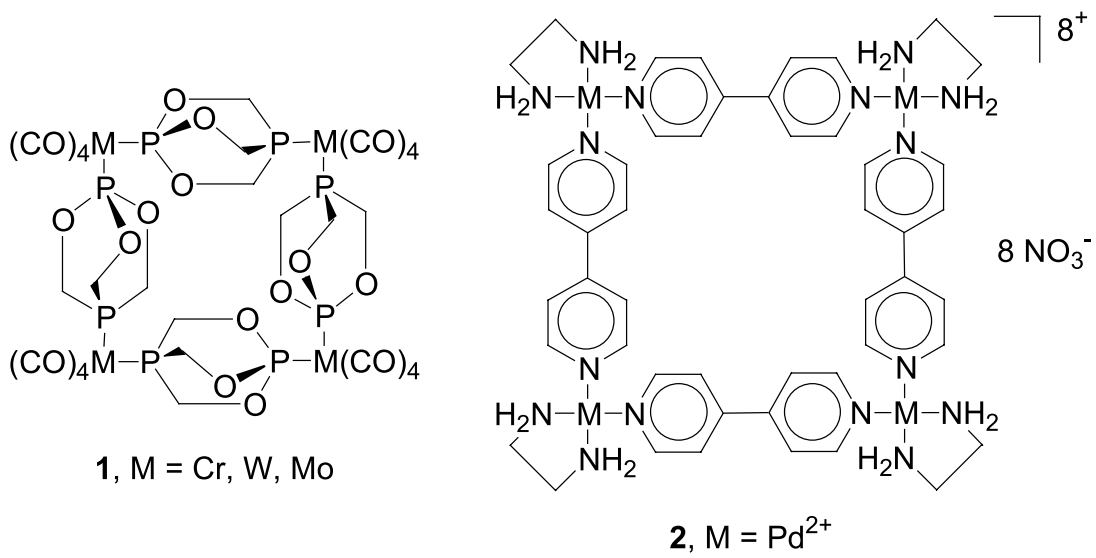

Fig. 1 Two of the earliest examples of tetrametallic molecular squares.

followed by the observation that for longer edges, closely 125 related square and triangle structures can coexist in dynamic equilibrium. The equilibrium is concentration- 126 dependent, with entropy favoring triangles, because they 127 assemble from fewer components. ${ }^{[6]}$ A related transfor- 128 mation, observed with a $\operatorname{Pd}(\mathrm{II})$ square and a pair of 129 dimeric $\mathrm{Pd}$ (II) loops in $\mathrm{D}_{2} \mathrm{O}$ as solvent, is formation of two 130 copies of a nonsquare catenated assembly. ${ }^{[7]}$ The driving 131 force for catenation is primarily the enhancement of 132 dispersion interactions accompanying van der Waals 133 contact of pairs of ligands. ${ }^{[8]}$ Formation of the catenated 134 species should occur most readily in solvents that offer 135 comparatively little stabilization via dispersion interactions. 136

Another early approach, since generalized to more than 137 70 different squares, triangles, prisms, rectangles, and 138 dodecahedra, also relies upon $\mathrm{Pd}(\mathrm{II})$ and $\mathrm{Pt}(\mathrm{II})$ as corners 139 but with chelating diphosphine ligands in place of 140 ethylenediamine to provide solubility and enforce subse- 141 quent cis coordination. ${ }^{[9]}$ The combination of corner units 142 with difunctional imine edges leads to octa-cationic 143 squares. The variety of ligand edges used ranges from 144 porphyrins, luminescent perylene diimides, ${ }^{[10]}$ and redox- 145 active ferrocene derivatives, ${ }^{[9]}$ to chiral linkers, such as 146 phosphine-functionalized binaphthols. ${ }^{[9]}$ With chiral 147 squares, one could easily envision applications such as 148 enantioselective catalysis, separation, or sensing. Indeed, 149 several examples of moderately enantioselective sensing 150 with a chiral-ligand-containing square were reported. ${ }^{[11]} 151$ Elaboration of squares via functionalization of the phos- 152 phine ligands is also possible. In one instance, squares 153 featuring pendent crown ethers were prepared. ${ }^{[9]}$

What about neutral squares? By using acetylides or 155 phenylides as edges, and forming metal(II)-carbon lin- 156 kages, squares lacking net charges can be formed. ${ }^{[12]} 157$ Other routes to neutral squares are described below.

\section{Assembly Principles}

Why has the square motif proven so popular in supramolecular coordination chemistry? First, with the right set of ancillary ligands, octahedral and square planar coordination geometries provide pairs of ligation sites oriented at $90^{\circ}$ with respect to each other. Second, square formation is typically accomplished with high efficiency, usually in a one-pot reaction. The one-pot formation process is often termed "self assembly," although a more accurate term might be "directed assembly,", which is the combination of cis coordination sites and edge-ligand rigidity, providing the necessary initial directions or instructions.

There is more to the process, however. In nearly every case, the square represents a thermodynamic rather than kinetic product. The reasons are several. The open ligation sites that characterize dimeric, trimeric, and noncyclic oligomeric species are temporarily filled by weakly coordinated solvent molecules; this, in turn, tends to keep these intermediates in solution. In addition, coordinatecovalent bonds tend to be labile, at least at elevated temperatures. Mistakes in the assembly process can be corrected, and assembly can continue until formation of the desired square molecule is complete (Fig. 2). ${ }^{[13]}$ If the completed tetrametallic cycles are less soluble than open oligomers (often the case), precipitation of the cycles can pull the reaction toward completion. Also favoring reaction completion (i.e., high yields) is the enthalpy released upon replacement of a weak metal-solvent bond with a somewhat stronger metal-ligand (edge) bond. For several reasons, therefore, the choice of solvent is important in square synthesis.

Ignoring occasional complications due to triangle formation, the directed assembly approach works well 158 with rigid difunctional ligands. What about flexible 


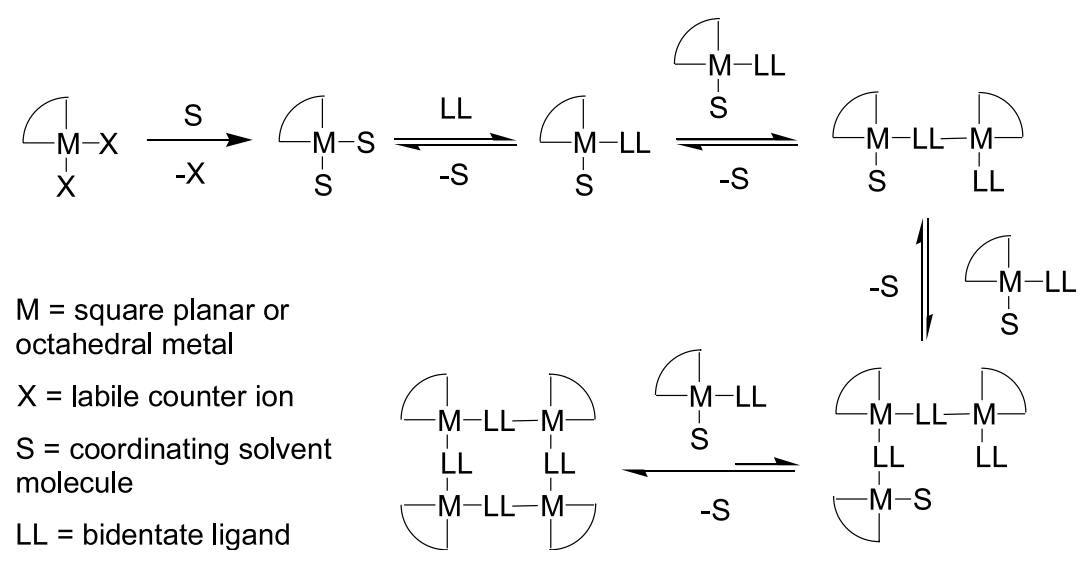

Fig. 2 Proposed mechanism of molecular square formation.

ligands? If the ligands are long, the standard assembly 168 then occupies the center of each edge, and the bent ligands approach tends to yield cyclic dimers instead of squares, 169 comprise the corners. One example is trans coordination consistent with simple entropy considerations. While no 170 of Pt(II) by 5,10-pyridyl-porphyrins, 3 (Fig. 3) ${ }^{[14]}$ Notaexamples were reported, presumably, in some cases, 171 bly, construction of the square in this way configures the templating methods could be used to generate flexible- 172 four porphyrin ligands in a coplanar fashion. The alwalled squares.

173 ternative assembly featuring linear ligand edges $(5,15$ -

174 pyridyl porphyrin species) and cis-coordinated metal ions

175 as corners configures alternating pairs of porphyrins in

176 a nominally cofacial arrangement, $4^{[9]}$ The two mo-

\section{Other Structural Motifs}

Squares can also be obtained by linearly coordinating bent 177 tifs obviously suggest different applications and dif(right-angle-containing) difunctional ligands. A metal ion 178 ferent ways of assembling multisquare structures.

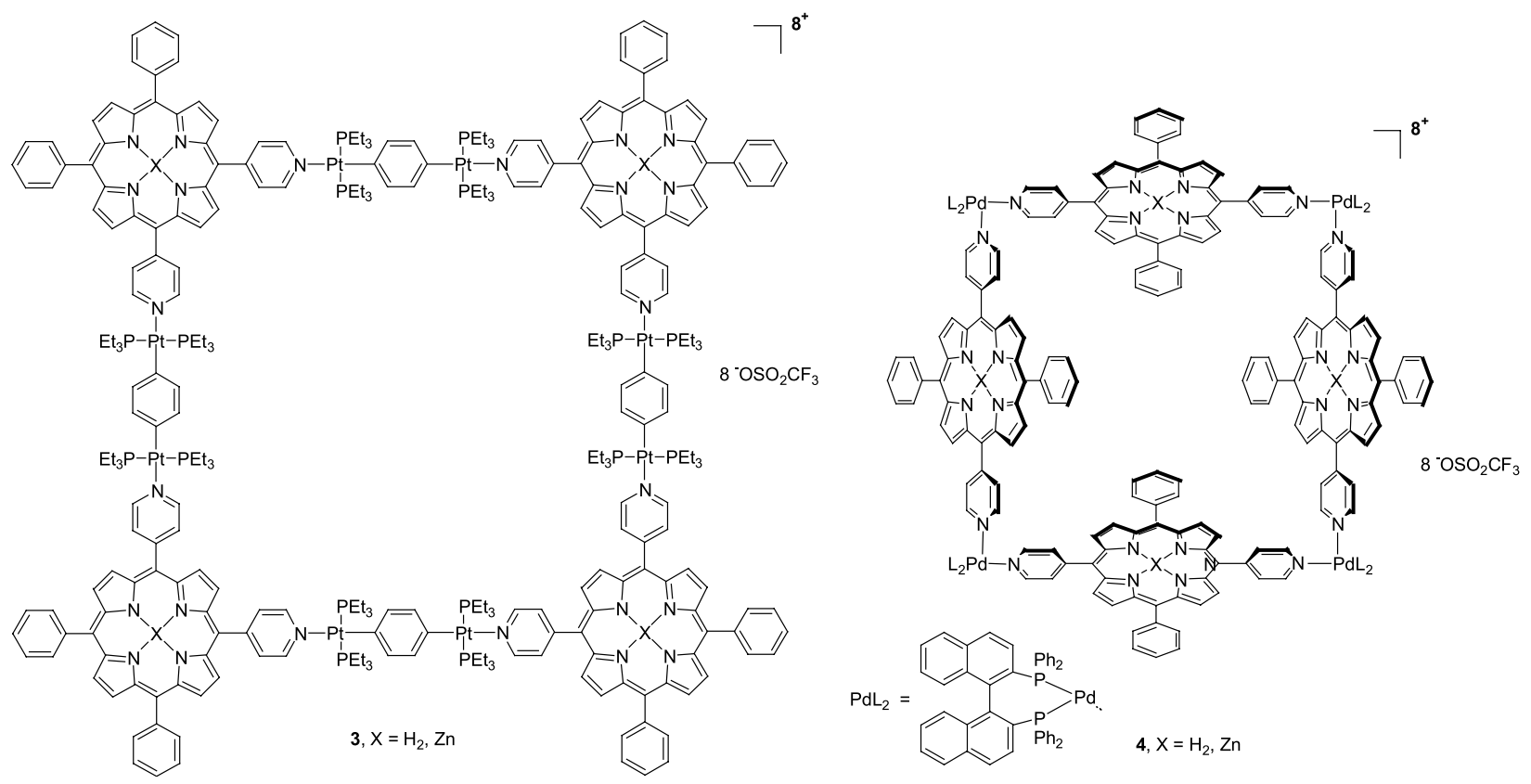

Fig. 3 Two examples of porphyrinic molecular squares. In 3, the cis orientation of pyridyl groups, combined with the trans binding of the Pt metal units, leads to a coplanar orientation for the four porphyrins. The trans configuration of dipyridyl porphyrins in $\mathbf{4}$ leads to a nominally cofacial arrangement of opposing pairs of porphyrins. For the particular example shown, however, steric demands force the porphyrins to fold in to yield an almost flat structure. 
Another alternative square motif is based on carbox- 231 ylate coordination of triply and quadruply bonded dime- 232 tallic corner units. ${ }^{[15]}$ The coordination geometry requires 233 the metal-metal bond axis to be oriented normal to the 234 plane of the square or, in some cases, triangle. An 235 interesting consequence is that monodentate ligand coor- 236 dination sites at the ends of the dimetallic units can often 237 be accessed, permitting squares to be linked "vertically", 238 in extended structures. Examples of squares based on 239 $\mathrm{Mo}_{2}{ }^{4+}, \mathrm{Ir}_{2}{ }^{4+}$, and $\mathrm{Rh}_{2}{ }^{4+}$ were reported. In solution, se- 240 veral show multistep metal redox reactivity that is 241 reversible on an electrochemical time scale. Unfortunate- 242 ly, on a longer time scale, the ease of oxidation together 243 with further chemical reactions renders some of the 244 squares structurally unstable in air or in oxygen-contain- 245 ing solutions.

A third alternative simply uses ferrocenes as walls in cyclophane-like structures. ${ }^{[16]}$ The ferrocenyl units typi- 247 cally are linked via flexible hydrocarbon chains appended to cyclopentadiene ligands. In other words, covalent 248 carbon-carbon bonds rather than coordinate-covalent 249 metal-nitrogen, -carbon, -oxygen, or-phosphorous bonds 250 are used.

\section{Porous Molecular Solids}

Remarkably, almost all molecular squares crystallize 255 as one-dimensional channel-containing materials, with 256 square cavities defining the channel width. For smaller 257 squares, microcrystallinity typically persists when the 258 squares are cast as thin films. For larger squares, such as 259 porphyrin squares, thin films are more typically amor- 260 phous. Films of both kinds, however, can display good 261 microporosity. Obviously, facilitating porosity for neutral 262 squares is the absence of potentially channel-blocking 263 counterions. Many charged squares, however, also feature 264 open channels with counterions positioned above and 265 below metal corners rather than within cavities. (An in- 266 teresting idea yet to be examined is that the cavity fields 267 generated by the spatially separated charges may engender 268 catalytic activity in a fashion reminiscent of many 269 reactions in zeolites.)

270

An important characteristic of molecular materials 271 based on squares is their ability to withstand solvent 272 removal without cavity collapse or loss of porosity. The 273 stability reflects the strength of coordinate-covalent bonds. 274 These bonds are often four to 10 times stronger than typical 275 hydrogen bonds.

Explored in most detail with respect to porosity were 277 molecular materials based on neutral tetrarhenium squares 278 $\left\{\left[\operatorname{Re}(\mathrm{CO})_{3} \mathrm{Cl}(\mu \text {-diimine })\right]_{4}\right.$ species $\}$ as described further 279 below. One step beyond strictly molecular materials are 280 ones composed of covalently or coordinatively linked 281 square units. Interesting examples here are the dimetallic- 282 cornered squares mentioned above, although their behavior as porous materials has yet to be explored. Another approach involves liquid-liquid interface polymerization. Acid-chloride functionalized linkers (short alkane chains) in one phase react with hydroxyl-functionalized porphyrin squares in another phase to generate high-porosity thinfilm polymers. ${ }^{[17]}$ Film growth is self-limiting (a few microns), and the density of pinhole defects that fully penetrate the films is low. A third approach uses phosphonate-functionalized porphyrin squares and makes use of the enormous affinity of $\mathrm{Zr}$ (IV) for phosphonates. Layer-by-layer porous film assembly can be done on glass, conductive glass, or ceramic platforms. The approach provides exceptional control over film thickness and yields films with channels strongly preferentially oriented in the direction normal to the platform. ${ }^{[18]}$

\section{Functional Squares}

Thin films of tetrarhenium squares $\left\{\left[\operatorname{Re}(\mathrm{CO})_{3} \mathrm{Cl}(\mu\right.\right.$-diimine) $]_{4}$ species $\}$ can function as molecular sieves, in the form of coatings on macroporous membrane supports or as overlayers on electrode surfaces. Sieving can be followed by spectrally observing the passage of dye molecules, via the coated membrane, from a reservoir solution to a receiving solution or by monitoring the electrochemical current produced by passage of a redoxactive probe molecule through a porous square coating. ${ }^{[19]}$ Sharp size cutoffs for probe molecules are observed, with the cutoffs corresponding to the sizes of cavities for isolated squares. Quantitative studies with films as thin as 20 nm show that molecular flux scales inversely with film thickness, demonstrating that rates of transport in these cases are limited by film-based diffusion rather than solution-to-film partitioning. For small- and mediumsized squares, known to form microcrystalline films, the observed molecular transport rates exceed by 20- to 50fold rates of transport through related amorphous metallopolymers featuring similar size cutoffs. These observations point to the importance of the one-dimensional channels created by alignment of squares and their cavities. Films of larger squares featuring metalloporphyrin walls are amenable to cavity functionalization via axial ligation of porphyrin-embedded metal ions. Extensions of this kind readily permit size cutoffs for film sieving to be rationally altered. In principle, chemical selectivity could also be engendered in this way.

Facilitated transport of sodium tosylate across a chloroform solution separating two aqueous phases was demonstrated. The carrier was a Pt(II)-bipyridine square featuring calixarene receptors as corner appendages. ${ }^{[9]}$

Advantage was taken of the luminescence of small- and medium-sized rhenium squares to accomplish chemical sensing: anions in solution ${ }^{[20]}$ and volatile aromatic 


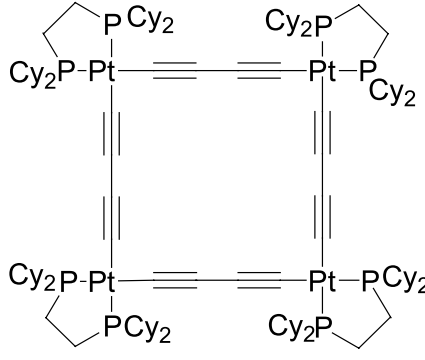

5

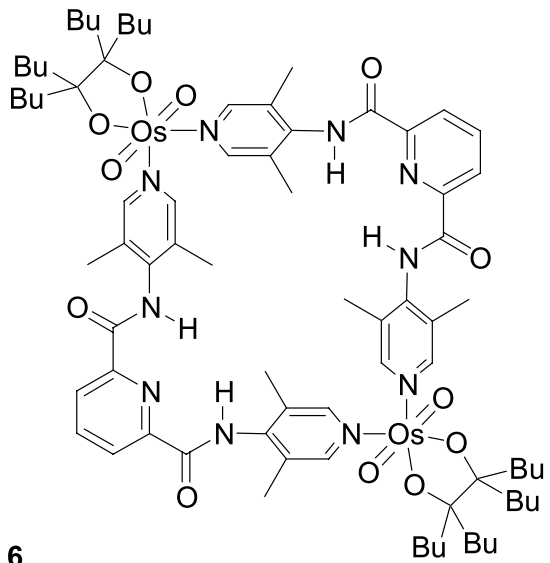

Fig. 4 Two examples of molecular squares designed for enhanced host-guest binding interactions. Structure $\mathbf{5}$ is able to bind $\mathrm{Ag}^{+}$ions between adjacent alkyne ligands via a $\pi$-tweezer effect. The corner receptor groups in $\mathbf{6}$ allow for enhanced binding of various amides through hydrogen-bonding contacts.

compounds in the vapor phase. ${ }^{[21]}$ Quartz crystal mi- 317 crogravimetry and modulated visible-light diffraction 318 schemes were also used to report on selective uptake of 319 analytes by porous molecular square films. ${ }^{[22]}$ By expand- 320 ing the number of potential host sites available, porous thin 321 films of squares offer sensitivity advantages over simple 322 monolayers of host compounds. Nevertheless, squares are 323 usually of limited effectiveness in sensing schemes. Few, 324 if any, candidate guest molecules (analytes) are square 325 shaped, ruling out efficient sensing based on shape com- 326 plementarity, and simple squares lack the requisite func- 327 tionalities to bind analytes in a highly chemically selective 328 fashion. (The examples described above depend on com- 329 paratively weak and nonspecific electrostatic, donor/ac- 330 ceptor, and dispersion interactions.)

Interesting exceptions are Pt-acetylide-based squares such as 5, that bind $\mathrm{Ag}^{+}$strongly based on specific 331 interactions with pairs of ethynyl groups (Fig. 4). ${ }^{[9,23]}$

Another involves a square featuring $\mathrm{OsO}_{4}$ units as two 332 diagonal corners and pyridine-2,6-dicarboxamides as the 333 other corners (6). This assembly selectively binds appro- 334 priately sized amides via multiple hydrogen-bonding 335 interactions. ${ }^{[24]}$

A potentially more general solution is to decorate the 337 interiors of large molecular squares with receptor ligands, 338 for example, by anchoring them to metal ions embedded 339 in porphyrins (Fig. 5). More than 100 cavity-modified 340 squares were described. Nevertheless, only a handful were 341 exploited for selective chemical sensing (e.g., iodine 342 sensing with tethered thiols, alkali metal ion sensing with 343 cavity-confined crown ethers, and zinc ion sensing with a 344 cavity-confined polyamine ligand). ${ }^{[25]}$

Encapsulation of metalloporphyrin-based epoxidation 346 catalysts by a porphyrin square was described. ${ }^{[26]}$ By 347 largely preventing the catalysts from destructively encountering other catalyst molecules, the square substantially extends catalyst lifetimes. At the same time, it creates a spatially restricted reaction environment that translates into substrate size selectivity in the epoxidation reaction. In one case, a dipyridylporphyrin catalyst was encapsulated within a $\mathrm{Zn}$ (II)-containing square, leaving two of the four porphyrinic zinc sites available for further ligation. By binding sterically demanding ligands to these sites, the substrate size selectivity was shown to be tunable. The degree of selectivity engendered in this way, however, is limited, because the putative cavity-modifying ligands are apparently sometimes bound to the square exterior.

\section{MOLECULAR BOXES AND CUBES}

In contrast to the vast assortment of molecular squares now in existence, relatively few examples of molecular boxes or cubes were synthesized, unless one views squares having tall edges (for example, porphyrinic squares) as open-ended flexible boxes. As defined here, molecular boxes are right-angle-containing complexes, where the metal coordination units are arranged in three dimensions, instead of two.

A ruthenium-based molecular cube was described. ${ }^{[27]}$ Comprising its corners are $\left\{\left([9] \mathrm{aneS}_{3}\right) \mathrm{Ru}\right\}^{2+}\left([9] \mathrm{aneS}_{3}=\right.$ 1,4,7-trithionane) units, linked by 4,4'-bipyridine edges. The assembly was obtained by reaction of stoichometric amounts of $\left\{\left([9] \mathrm{aneS}_{3}\right) \mathrm{Ru}\right\}^{2+}$ and $4,4^{\prime}$-bpy (8:12) in a noncoordinating solvent for 4 weeks. Key to the cube synthesis is the sulfurcontaining macrocycle. By occupying three coordination sites in a facial fashion, it leaves 

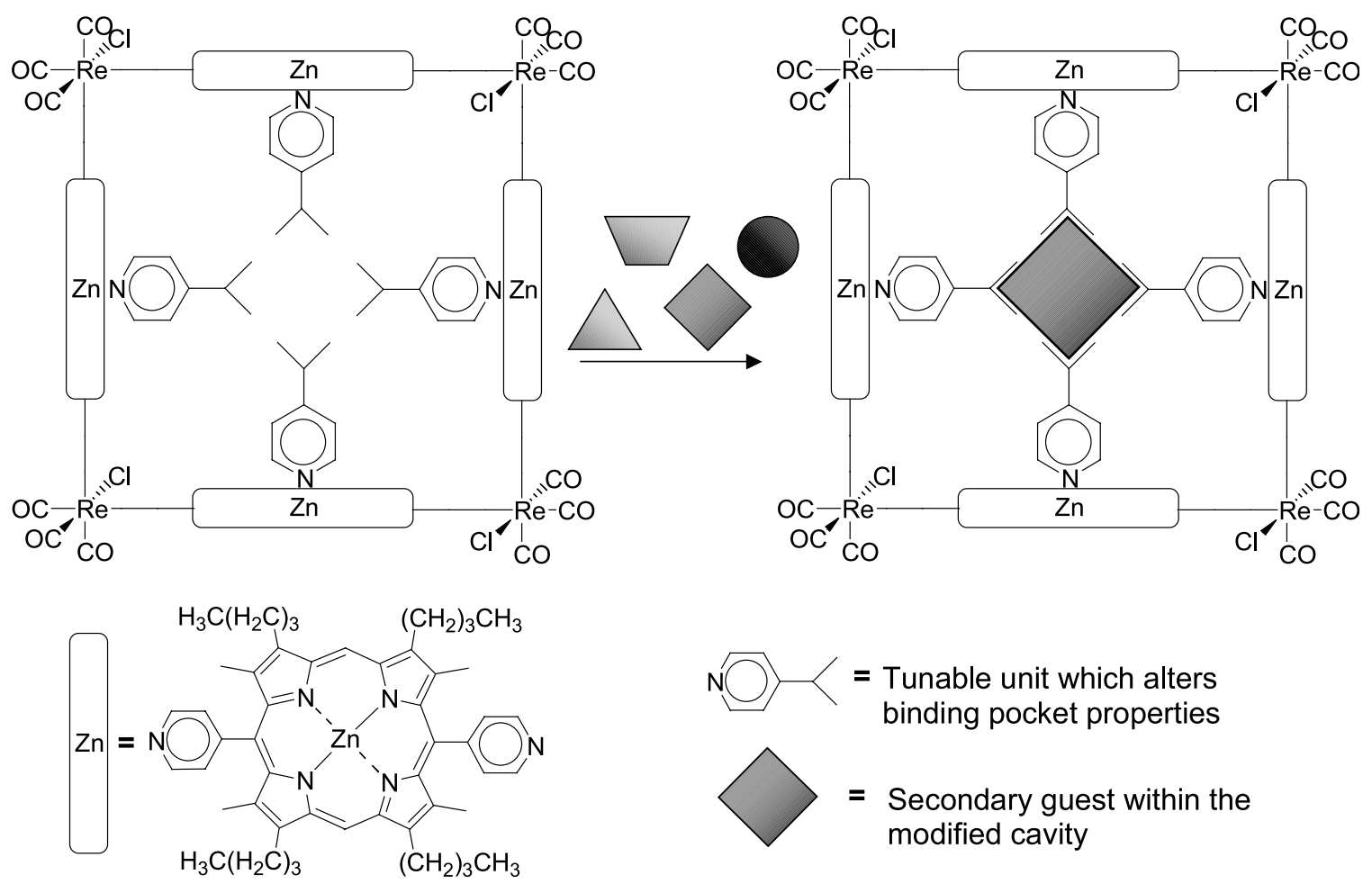

Fig. 5 Porphyrin square functionalization for selective guest binding. (Go to www.dekker.com to view this figure in color.)

open three additional sites (also facially arranged) for 374 ligation of the cube's rigid edges. A crystal structure of a 375 cube fragment, $\left\{\left([9] \mathrm{aneS}_{3}\right)\left(4,4^{\prime}-\mathrm{bpy}\right)_{3} \mathrm{Ru}\right\}^{2+}$, clearly illus- 376 trates the anticipated mutually perpendicular arrangement 377 of the three bpy ligands. Although an x-ray crystal 378 structure of the cube was not reported, ${ }^{1} \mathrm{H}-\mathrm{NMR}$ of the 379 sample reveals two sets of 4,4'-bpy resonances, consistent 380 with the formation of the highly symmetrical product. 381

Two types of molecular boxes featuring (ethylenedia- 382 mine)Pd(II) corners and polypyridyl linkers were de- 383 scribed. ${ }^{[28,29]}$ One uses roughly rectangular tetrapyridyl 384 ligands as walls for an open-ended cube. (7) Multiple 385 isomers were obtained, and one is shown in Fig. 6. By 386 carrying out the synthesis in the presence of an appropri- 387 ate guest molecule, the isomer distribution can be 388 controlled, as can an equilibrium between competing 389 prism and cube assemblies. The second type comprises a 390 family of elongated boxes. The walls here consist of 3,5- 391 linked terpyridine, tetrapyridine, and pentapyridine 392 ligands with 6,8 , or 10 (en)Pd(II) units serving as 393 connectors along the box seams. Remarkably, box 394 formation is efficiently templated by rod-like guest 395 molecules. In the absence of templates, either no 396 supramolecular complex formation is seen, or a range of 397 complexes (trimers, tetramers, and pentamers) are present, 398 presumably in dynamic equilibrium. These observations 399 clearly point toward the idea of employing dynamic combinatorial libraries to generate optimal supramolecular inorganic hosts for specific molecular guests. In addition to x-ray crystallography and solution-phase NMR spectroscopy, cold-spray ionization mass spectroscopy was shown to be useful for identifying reaction products, including thermodynamically unstable products in these initial synthesis studies.

A series of mixed-metal molecular cubes, based on the coordination chemistry of Prussian blue and other polymeric cyanometalates, was described. The cubes feature $\mathrm{Rh}$ and either Co or Mo on alternating corners, with edges consisting of bridging $\mathrm{CN}$ groups. ${ }^{[30]}$ The cubes are formed by the binary combination of molecular squares, subsequent to removal of axially bound chloride ligands. The intermediate molecular squares species are formed by the reaction of $\left[C p^{*} M(\mathrm{CN})_{3}\right]^{-}\left(C p^{*}=\right.$ pentamethyl cyclopentadienyl, $M=\mathrm{Rh}, \mathrm{Co})$ with either $\left\{\left[(\mathrm{R}) M \mathrm{Cl}_{2}\right]_{2}\right\}(R=$ $\left.\mathrm{Cp}^{*}, \mathrm{C}_{10} \mathrm{H}_{14} ; M=\mathrm{Rh}, \mathrm{Ru}\right)$ or $\left(\eta^{6}-\mathrm{C}_{6} \mathrm{H}_{3} \mathrm{Me}_{3}\right) \mathrm{Mo}(\mathrm{CO})_{3}$. The $C p^{*}$ ligands serve to block three of the six octahedral sites, thereby precluding polymer formation. Recently, however, the controlled formation of a double cube (one shared corner) was described. ${ }^{[31]}$

An interesting property of cyanide-bridged molecular cubes is their ability to bind alkali metals (especially $\mathrm{K}^{+}$ and $\mathrm{Cs}^{+}$) selectively within the cube interior. ${ }^{[30]}$ 

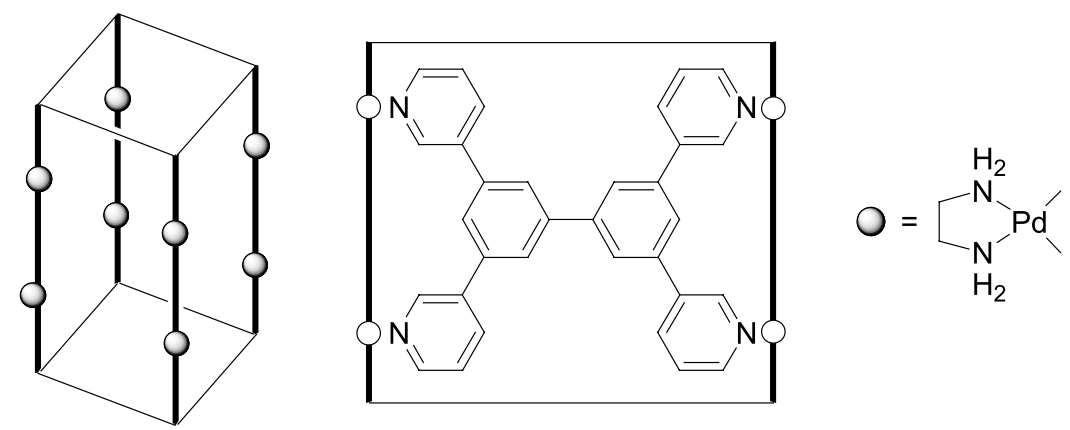

Fig. 6 One of three isomeric boxes that can be obtained from the combination of 3,3',5,5'-tetrakis(3-pyridyl)biphenyl and (en) $\mathrm{Pd}\left(\mathrm{NO}_{2}\right)_{2}$.

Crystallographic analysis of a Rh-Mo molecular cube 433 indicates bond formation between the cationic guest and 434 the cube's CN edges. When $\mathrm{K}^{+}$is encapsulated, the ion is 435 distributed over two equivalent positions, both slightly displaced from the cube center. Encapsulated cesium ions, on the other hand, occupy a central position. The locational differences reflect differences in ionic radii: 2.02 and $1.78 \AA$ for $\mathrm{Cs}^{+}$and $\mathrm{K}^{+}$, respectively, with coordination numbers of 12 for each. The structural differences translate into a nearly 4000-fold preference for uptake of $\mathrm{Cs}^{+}$ versus $\mathrm{K}^{+}$from salt solutions, where the difference was evaluated competitively via ${ }^{133} \mathrm{Cs}-\mathrm{NMR}$ measurements.

\section{CONCLUSION}

\author{
436
}

437 ously, further advances in molecular and higher-order assembly chemistry will facilitate these developments and likely stimulate others.

\section{CROSS-REFERENCES}

Classification and Nomenclature of Supramolecular Compounds; Mesoporous Materials; Nanotechnology; SelfAssembly: Definition and Kinetic and Thermodynamic Considerations; Supramolecular Catalysis; Supramolecular Chemistry, Definition; Supramolecular Chemistry, Historical Basis; Supramolecular Chirality; Supramolecular Electrochemistry; Supramolecular Photochemistry; Supramolecular Synthons.

\section{REFERENCES}

1. Odell, B.; Reddington, M.V.; Slawin, A.M.Z.; Spencer, N.; Stoddart, J.F.; Williams, D.J. Cyclobis(paraquat-p-phenylene), a tetracationic general-purpose receptor. Angew. Chem. 1988, 100 (11), 1605-1608.

2. Eddaoudi, M.; Li, H.; Yaghi, O.M. Highly porous and stable metal-organic frameworks. Structure design and sorption properties. J. Am. Chem. Soc. 2000, 122 (7), 1391-1397.

3. Stricklen, P.M.; Volcko, E.J.; Verkade, J.G. Novel homo and heterometallic coordination macrocycles. J. Am. Chem. Soc. 1983, 105 (8), 2494-2495.

4. Fujita, M.; Yazaki, J.; Ogura, K. Preparation of a macrocyclic polynuclear complex, [(en)Pd(4,4'bpy)]4(NO3)8 (en=ethylenediamine, bpy=bipyridine), which recognizes an organic molecule in aqueous media. J. Am. Chem. Soc. 1990, 112 (14), 5645-5647.

5. Fujita, M.; Yazaki, J.; Ogura, K. Macrocyclic polynuclear complexes $\left[(\mathrm{en}) \mathrm{M}\left(4,4^{\prime}\right.\right.$-bpy $\left.)\right] 4(\mathrm{NO} 3) 8 \quad(\mathrm{M}=$ palladium or 
platinum) as "inorganic cyclophane." Their ability for 514 molecular recognition. Tetrahedron Lett. 1991, 32 (40), 515 5589-5592.

6. Fujita, M.; Sasaki, O.; Mitsuhashi, T.; Fujita, T.; Yazaki, 517 J.; Yamaguchi, K.; Ogura, K. On the structure of transition- 518 metal-linked molecular squares. Chem. Commun. 1996, 519 (13), 1535-1536.

7. Fujita, M.; Ibukuro, F.; Yamaguchi, K.; Ogura, K. A 521 molecular lock. J. Am. Chem. Soc. 1995, 117 (14), 4175- 522 4176.

8. Hori, A.; Kumazawa, K.; Kusukawa, T.; Chand, D.K.; 524 Fujita, M.; Sakamoto, S.; Yamaguchi, K. DOSY study on 525 dynamic catenation: Self-assembly of a [3]catenane as a 526 meta-stable compound from twelve simple components. 527 Chem.-Eur. J. 2001, 7 (19), 4142-4149.

9. Stang, P.J.; Olenyuk, B. Self-assembly, symmetry, and 529 molecular architecture: Coordination as the motif in the 530 rational design of supramolecular metallacyclic polygons 531 and polyhedra. Acc. Chem. Res. 1997, 30 (12), 502-518. 532

10. Wurthner, F.; Sautter, A. Highly fluorescent and electro- 533 active molecular squares containing perylene bisimide 534 ligands. Chem. Commun. 2000, (6), 445-446.

1. Lee, S.J.; Lin, W. A chiral molecular square with metallo- 536 corners for enantioselective sensing. J. Am. Chem. Soc. 537 2002, 124 (17), 4554-4555.

12. Leininger, S.; Olenyuk, B.; Stang, P.J. Self-assembly of 539 discrete cyclic nanostructures mediated by transition 540 metals. Chem. Rev. 2000, 100 (3), 853-907. 541

13. Davis, A.V.; Yeh, R.M.; Raymond, K.N. Supramolecular 542 assembly dynamics. Proc. Natl. Acad. Sci. U. S. A. 2002, 543 99 (8), 4793-4796.

14. Drain, C.M.; Lehn, J.-M. Self-assembly of square multi- 545 porphyrin arrays by metal ion coordination. J. Chem. Soc., 546 Chem. Commun. 1994, (19), 2313-2315.

15. Cotton, F.A.; Lin, C.; Murillo, C.A. Supramolecular arrays 548 based on dimetal building units. Acc. Chem. Res. 2001, 34549 (10), 759-771.

16. Beer, P.D.; Gale, P.A. Anion recognition and sensing: The 551 state of the art and future perspectives. Angew. Chem., Int. 552 Ed. Engl. 2001, 40 (3), 486-516.

17. Keefe, M.H. Ph.D. Dissertation; Northwestern University, 554 2001.

18. Wightman, M. M.S. Thesis; Northwestern University, 2001. 556

19. Williams, M.E.; Hupp, J.T. Scanning electrochemical 557 microscopy assessment of rates of molecular transport 558 through thin films of mesoporous films of porphyrinic 559 'molecular squares.' J. Phys. Chem., B 2001, 105 (37), 560 8944-8950.

20. Slone, R.V.; Yoon, D.I.; Calhoun, R.M.; Hupp, J.T. 562 Luminescent rhenium/palladium square complex exhibit- 563 ing excited state intramolecular electron transfer reactivity and molecular anion sensing characteristics. J. Am. Chem. Soc. 1995, 117 (47), 11813-11814.

21. Sun, S.-S.; Lees, A.J. Self-assembly triangular and square rhenium(I) tricarbonyl complexes: A comprehensive study of their preparation, electrochemistry, photophysics, photochemistry, and host-guest properties. J. Am. Chem. Soc. 2000, 122 (37), 8956-8967.

22. Keefe, M.H.; Slone, R.V.; Hupp, J.T.; Czaplewski, K.F.; Snurr, R.Q.; Stern, C.L. Mesoporous thin films of "molecular squares" as sensors for volatile organic compounds. Langmuir 2000, 16 (8), 3964-3970.

23. Alqaisi, S.M.; Galat, K.J.; Chai, M.H.; Ray, D.G.; Rinaldi, P.L.; Tessier, C.A.; Youngs, W.J. Synthesis of neutral tetranuclear and octanuclear macrocyclic platinum-butadiyne heterocyclynes. J. Am. Chem. Soc. 1998, 120 (46), 12149-12150.

24. Jeong, K.-S.; Cho, Y.L.; Song, J.U.; Chang, H.-Y.; Choi, M.-G. Neutral macrocyclic boxes spontaneously assembled from osmium tetraoxide, olefin, and pyridyl ligand. J. Am. Chem. Soc. 1998, 120 (42), 10982-10983.

25. Mines, G.A.; Tzeng, B.-C.; Stevenson, K.J.; Li, J.; Hupp, J.T. Microporous supramolecular coordination compounds as chemosensory photonic lattices. Angew. Chem., Int. Ed. Engl. 2002, 41 (1), 154-157.

26. Merlau, M.L.; Mejia, M.d.P.; Nguyen, S.T.; Hupp, J.T. Artificial enzymes formed through directed assembly of molecular square encapsulated epoxidation catalysts. Angew. Chem., Int. Ed. Engl. 2001, 40 (22), 4239-4242.

27. Roche, S.; Haslam, C.; Heath, S.L.; Thomas, J.A. Selfassembly of a supramolecular cube. Chem. Commun. 1998, (16), 1681-1682.

28. Yamanoi, Y.; Sakamoto, Y.; Kusukawa, T.; Fujita, M.; Sakamoto, S.; Yamaguchi, K. Dynamic assembly of coordination boxes from (en)Pd(II) unit and a rectangular panel-like ligand: NMR, CSI-MS, and x-ray studies. J. Am. Chem. Soc. 2001, 123 (5), 980-981.

29. Aoyagi, M.; Biradha, K.; Fujita, M. Quantitative formation of coordination nanotubes templated by rodlike guests. J. Am. Chem. Soc. 1999, 121 (32), 7457-7458.

30. Klausmeyer, K.K.; Wilson, S.R.; Rauchfuss, T.B. Alkali metal-templated assembly of cyanometalate "Boxes", (NEt4)3\{M[Cp*Rh(CN)3]4[Mo(CO)3]4\} (M=K, Cs). Selective binding of Cs+. J. Am. Chem. Soc. 1999, 121 (12), 2705-2711.

31. Contakes, S.M.; Kuhlman, M.L.; Ramesh, M.; Wilson, S.R.; Rauchfuss, T.B. Systematic assembly of the double molecular boxes: $\{\mathrm{CsC}[\mathrm{CpCo}(\mathrm{CN}) 3] 4[\mathrm{Cp} * \mathrm{Ru}] 3\}$ as a tridentate ligand. Proc. Natl. Acad. Sci. U. S. A. 2002, 99 (8), 4889-4893. 